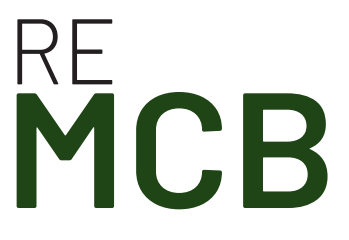

Revista Ecuatoriana de Medicina y Ciencias Biológicas

Volumen 42. No. 1, Mayo 2021

\title{
Caracterización molecular de genes de resistencia a $\beta$-lactámicos en aislados bacterianos clínicos de la familia Enterobacteriaceae
}

\author{
Molecular characterization of $\beta$-lactam resistance genes in clinical bacterial \\ isolates of the Enterobacteriaceae family
}

\author{
Duncan Coral'1, María Fernanda Yauri1* e lliana Alcocer'
}

\begin{abstract}
'Laboratorio de Microbiología, Escuela de Ciencias Biológicas, Pontificia Universidad Católica del Ecuador, Quito, Ecuador.
\end{abstract}

*Autor de correspondencia: mfyaurib@puce.edu.ec

Recibido 8-01-2021

Aceptado 17-04-2021

DOI: $10.26807 /$ remcb.v42i1.886

e-ISSN 2477-9148

๑) 2021. Este es un artículo publicado en acceso abierto bajo una licencia CC BY 4.0

Como citar este artículo: Coral D, Yauri MF, Alcocer I. 2021 Caracterización molecular de genes de resistencia a $\beta$-lactámicos en aislados bacterianos clínicos de la familia Enterobacteriaceae. Revista Ecuatoriana de Medicina y Ciencias Biológicas 42(1): 63-77. doi: 10.26807/remcb.v42i1.886
Resumen.- Las infecciones desarrolladas por enterobacterias productoras de $\beta$-lactamasas de espectro extendido (BLEE) se relacionan con altas tasas de mortalidad y morbilidad en ambientes hospitalarios, gracias a su capacidad de hidrolizar antibióticos $\beta$-lactámicos. El objetivo del presente estudio fue caracterizar los genes que confieren resistencia a $\beta$-lactámicos en enterobacterias obtenidas de un hospital de tercer nivel de la ciudad de Quito. Se colectaron 153 enterobacterias y se identificó la especie mediante pruebas bioquímicas establecidas. El estudio seleccionó los aislados que presentaron producción de enzimas BLEE analizado por el método de sinergia de doble disco y el sistema automatizado VITEK 2 proporcionado en el hospital. De los 22 aislados seleccionados, 19 fueron identificados como Escherichia coli y 3 como Klebsiella oxytoca. La identificación de genes de resistencia empleó reacción en cadena de la polimerasa y usó cebadores específicos de cada gen codificante de BLEE (bla $a_{\mathrm{CTX}-\mathrm{M}^{\prime}}$ bla $a_{\mathrm{TEM}}$ bla $\left.a_{\mathrm{SHV}}\right)$ y de enzimas carbapenemasas $\left(b / a_{\mathrm{KPC}}, b / a_{\mathrm{IMP},}, b / a_{\mathrm{VIM}^{\prime}} b / a_{\mathrm{NDM}}\right)$. La identificación de la variante alélica reportó que 11/22 aislados presentaron el gen bla $a_{\text {CTX-M-15 }}$ y $4 / 22$ el gen bla $a_{\text {TEM-1 }}$. Ninguno aislado presentó genes de resistencia a carbapenémicos. La tipificación molecular se realizó con electroforesis en Gel de Campo Pulsado y mostró ausencia de diseminación clonal.

Palabras clave: $\beta$-lactamasas de espectro extendido (BLEE), enterobacterias, genes de resistencia, resistencia bacteriana, variante alélica.

Abstract.- Infections developed by extended spectrum $\beta$-lactamase-producing Enterobacteriaceae (ESBL) are associated with high mortality and morbidity rates in hospital settings due to their ability to hydrolyze $\beta$-lactam antibiotics. The objective of this study was to characterize the genes that confer resistance to $\beta$-lactams in Enterobacteriaceae obtained from a tertiary hospital in the city of Quito. 153 members of the Enterobacteriaceae were collected and the species was identified with biochemical tests. The study selected the isolates that presented ESBL enzyme production analyzed by the double disk synergy method and the VITEK 2 automated system provided by the hospital. Of the 22 selected isolates, 19 were identified as Escherichia coli and 3 as Klebsiella oxytoca. The identification of resistance genes used a polymerase chain reaction and used specific primers for each gene encoding ESBL (b/a $a_{C T X-M^{\prime}}$ $\left.b / a_{\mathrm{TEM}}, b / a_{\mathrm{SHV}}\right)$ and carbapenemase enzymes $\left(b / a_{\mathrm{KPC}}, b / a_{\mathrm{IMP}} b / a_{\mathrm{VIM}}, b / a_{\mathrm{NDM}}\right)$. The identification of the allelic variant reported that $11 / 22$ isolates presented the bla ${ }_{\text {CTX-M-15 }}$ gene and 4/22 the bla $a_{\text {TEM-1 }}$ gene. None of the isolates presented genes for resistance to carbapenems. Molecular typing was performed with Pulsed Field Gel Electrophoresis and showed the absence of clonal spread.

Keywords: allelic variant, bacterial resistance, Enterobacteriaceae, extended spectrum betalactamases (ESBL), resistance genes. 


\section{Introducción}

La familia Enterobacteriaceae comprende más de 30 géneros y alrededor de 100 especies bacterianas. Los miembros de esta familia son bacterias Gram negativas, de forma bacilar y están presentes en el intestino de humanos y otros animales homeotermos formando parte de la microbiota natural (CDC 2013). Esta familia alberga géneros de importancia clínica como: Escherichia, Shigella, Klebsiella, Enterobacter, Proteus, Salmonella y Citrobacter. Las especies patógenas producen infecciones oportunistas, en especial a pacientes hospitalizados con sistemas inmunitarios comprometidos como pacientes con VIH, pacientes oncológicos, niños y ancianos (Adeolu et al. 2016).

En la actualidad, la eficacia de estos agentes se ha reducido por el desarrollo de diversos mecanismos de resistencia por parte de la bacteria. Así tenemos: 1) producción de enzimas tipo $\beta$-lactamasas que inactivan los antibióticos por hidrólisis; 2) modificación del sitio blanco de acción, disminuyendo la afinidad de unión del antibiótico a la bacteria; 3) cierre de canales porinas, disminuyendo la permeabilidad de la célula bacteriana al antibiótico, y 4) bombas activas que extraen el antibiótico desde el interior de la célula al exterior (Zeng y Lin 2013, Cho et al. 2014, Shaikh et al. 2015).

Las $\beta$-lactamasas son enzimas producidas por bacterias Gram positivas y Gram negativas. En este grupo se encuentran las $\beta$-lactamasas de espectro extendido (BLEE), enzimas codificadas por genes cromosómicos y plasmidiales. Estas enzimas comprenden $\beta$-lactamasas tipo SHV, TEM, CTX-M, Amp-C y PER (D’Andrea et al. 2013, López et al. 2016).

Entre las $\beta$-lactamasas de espectro extendido (BLEE), el tipo CTX-M es el grupo de mayor importancia clínica en la actualidad. La mayoría de estudios que involucran bacterias productoras de BLEE lo reportan (Brolund y Sandegren 2016). El grupo CTX-M fue descrito por primera vez a partir de un aislado de Escherichia coli en el año 1989 en Munich, Alemania. Debe su nombre a la capacidad de producir la enzima cefotaximasa, enzima que confiere resistencia a la cefotaxima (Cantón et al. 2012). Se originó a partir de genes cromosomales presentes en miembros del género Kluyvera. Este género de bacterias comprende varias especies ambientales y no suelen estar relacionadas a reportes clínicos (Rossolini et al. 2008, Bevan et al. 2017).

La familia CTX-M se divide en 6 grupos: CTX-M-1, CTX-M-2, CTX-M-9, CTX-M-8, CTX-M-25 y KLUC, con 210 variantes reportados actualmente (Ding et al. 2018). El grupo CTX-M-1 y el CTX-M-9 son los que tienen una mayor distribución a nivel mundial, siendo la variante CTX-M-15 (del grupo CTX-M-1), la de mayor prevalencia (Bevan et al. 2017, Ding et al. 2018, Rivoarilala et al. 2018).

Las enzimas BLEE del tipo TEM fueron identificadas por primera vez en una E. coli, en Atenas (Grecia) en 1965. Estas enzimas fueron ampliamente reportadas en casos de resistencia a $\beta$-lactámicos entre bacterias Gram negativas a finales de los años 90s. En la actualidad, se han reportado 180 variantes de esta enzima, siendo las variantes TEM-1 y TEM-2 las que tienen mayor distribución a nivel mundial. Estas enzimas, son responsables de resistencia a penicilina, ampicilina y cefalosporinas de primera generación (López et al. 2016).

Un grupo importante de antibióticos $\beta$-lactámicos lo constituyen los carbapenémicos. Este tipo de antibióticos cuenta con un mayor espectro de actividad y con una mayor resistencia a las enzimas $\beta$-lactamasas. Por esta razón, son empleados como tratamientos alternativos eficaces en casos de infecciones producidas por bacterias multirresistentes. Su mecanismo de acción es similar al de $\beta$-lactámicos tradicionales, pero su efectividad y estabilidad son mucho mayores (Doi et al. 2017). Esta propiedad lo confiere la presencia de un carbono en la posición C-1 y a la configuración trans del anillo $\beta$-lactámico en los carbonos C-5 y C-6, incrementando su estabilidad frente a enzimas $\beta$-lactamasas (Zeng y Lin 2013).

La resistencia mediada por enzimas se ha incrementado en los últimos años, convirtiéndose en uno de los problemas más graves que la salud pública mundial debe afrontar. En el informe de la Organización Mundial de la Salud del 28 de enero de 2018 se reconoce que esta problemática es una realidad en cualquier parte del mundo. El problema se agrava al considerar que muchas 
enterobacterias son resistentes a múltiples drogas (OMS 2018). El Ecuador no es ajeno a esta realidad y desde el año 2010 se ha reportado un incremento de la resistencia bacteriana (Zurita 2013). Datos del Instituto Nacional de Estadísticas y Censos (INEC) muestran que, en el año 2014, 30269 personas fueron diagnosticadas con enfermedades relacionadas con infecciones bacterianas (INEC 2017).

\section{Materiales y Métodos}

Población de estudio.- Los aislados bacterianos fueron obtenidos de un centro hospitalario de tercer nivel de la ciudad de Quito, entre los meses de octubre del 2016 a mayo del 2017. El hospital proporcionó únicamente aislados clínicos de la familia Enterobacteriaceae. El total de aislados colectados fue de 153, de los cuales 22 fueron seleccionados por la producción enzimática de $\beta$-lactamasas de espectro extendido (BLEE), previamente identificado por la casa de salud. Los aislados obtenidos fueron llevados al Laboratorio de Microbiología de la Pontificia Universidad Católica del Ecuador (PUCE), a través del uso de medios de transporte Stuart y medidas de bioseguridad adecuadas. En el laboratorio, los aislados bacterianos fueron procesadas, incorporados y codificados a la Colección Bacteriana - Quito Católica (CB-QCA).

Identificación fenotípica.- La identificación de cada aislado proporcionado por el centro hospitalario se realizó a través de una batería de pruebas bioquímicas establecidas que comprenden el uso de varios medios diferenciales. Los medios de cultivo utilizados en este estudio fueron manufacturados por la empresa BD Difco ${ }^{\text {TM }}$ y fueron: agar Citrato de Simmons, agar Triple Azúcar y Hierro (TSI), agar Motilidad-Indol-Lisina (MIL), agar Motilidad-Indol-Ornitina (MIO), caldo Rojo de Metilo y Vogues Proskauer (RMNP), agar fenilalanina y agar urea (BD Diagnostics 2009).

Obtención de perfiles fenotípicos de resistencia.- El centro hospitalario proporcionó, junto a los aislados bacterianos, los perfiles de resistencia obtenidos a través del sistema automatizado VITEK $2^{\text {TM }}$. Las tarjetas empleadas en la determinación de la concentración inhibitoria mínima (CIM) fueron AST-N272 (bioMérieux, Inc.). En el laboratorio de Microbiología PUCE se realizaron análisis de sensibilidad a antimicrobianos empleando el método de difusión en disco KirbyBauer (Bauer et al. 1966, CLSI 2018). Los discos de antibióticos utilizados en la determinación de la sensibilidad antibiótica fueron: Ampicilina $(10 \mu \mathrm{g})$, Ampicilina/Sulbactam $(10 \mu \mathrm{g} / 10 \mu \mathrm{g})$, Piperacilina/tazobactam $(100 \mu \mathrm{g} / 10 \mu \mathrm{g})$, Cefazolina $(30 \mu \mathrm{g})$, Cefoxitina $(30 \mu \mathrm{g})$, Ceftriaxona (30 $\mu \mathrm{g})$, Cefepima $(30 \mu \mathrm{g})$, Gentamicina $(10 \mu \mathrm{g})$, Tobramicina $(10 \mu \mathrm{g})$, Amikacina $(30 \mu \mathrm{g})$, Ácido nalidíxico $(30 \mu \mathrm{g})$, Norfloxacina $(10 \mu \mathrm{g})$, Ciprofloxacina $(5 \mu \mathrm{g})$, Levofloxacina $(5 \mu \mathrm{g})$, Trimetoprima/ sulfametoxazol $(25 \mu \mathrm{g})$ y Aztreonam $(30 \mu \mathrm{g})$. La interpretación de los halos de inhibición obtenidos siguió los lineamientos establecidos por el Instituto de Estándares Clínicos y de Laboratorio, CLSI (2018).

Identificación fenotípica de $\beta$-lactamasas de espectro extendido (BLEE). - Para la identificación fenotípica de los aislados productores de BLEE se empleó el Método de sinergia de doble disco. En una placa de MHA se inoculó una suspensión bacteriana al 0,5 de la escala de McFarland. El ensayo empleó discos de Cefotaxima $(5 \mu \mathrm{g})$, Cefotaxima/Ácido Clavulánico $(30 \mu \mathrm{g} / 10 \mu \mathrm{g})$, Ceftazidima $(30 \mu \mathrm{g})$ y Ceftazidima/Ácido Clavulánico $(30 \mu \mathrm{g} / 10 \mu \mathrm{g})$ que fueron colocados de manera equidistante. Después de incubar a $37^{\circ} \mathrm{C}$ por 18 horas, se midió el diámetro de los halos de inhibición obtenidos. Para la interpretación de resultados, si el halo de inhibición del antibiótico más el inhibidor es $5 \mathrm{~mm}$ mayor al del antibióticos sin inhibidor se reporta como una prueba positiva a la producción de BLEE (CLSI 2018).

Identificación fenotípica de carbapenemasas. - La identificación fenotípica de carbapenemasas usó el Triton Hodge Test (THT) que empleó TritonX-100. Para ello, se eliminó la humedad de la superficie de una placa de agar Mueller Hinton (MHA) estéril y se agregó $50 \mu \mathrm{L}$ de TritonX-100 en la superficie de la placa de MHA. El TritonX-100 fue distribuido con un cotonete estéril sobre toda la superficie de la placa hasta su completa absorción. Posterior a este procedimiento, se preparó una suspensión de 0,5 en la escala de McFarland, en caldo Mueller Hinton, de la cepa ATCC 25922 de Escherichia coli. A continuación, la suspensión fue sembrada en la placa de MHA previamente impregnada de TritonX-100. Una vez inoculada la placa, se colocó un disco de 
meropenem $(10 \mu \mathrm{g})$ en el centro de ésta. Con ayuda de un cotonete estéril, se inoculó en línea recta el microorganismo problema, el cual partió del extremo del disco al borde de la placa. Este proceso se repitió en 2 ocasiones, una con la cepa control positiva (K. pneumoniae ATCC BAA 1705) y otra con la cepa control negativa (K. pneumoniae ATCC BAA 1706). Finalmente, las placas fueron incubadas a $35^{\circ} \mathrm{C}$ por 16 - 18 horas. Para la interpretación de los resultados, se observó la interacción entre el organismo analizado y la cepa ATCC 25922 de E. coli. Un resultado positivo demuestra un sobrecrecimiento de la cepa indicadora hacia el disco del carbapenem empleado en la intersección de la estría con la zona de inhibición. Un resultado negativo se interpretó cuando la zona de inhibición permanece inalterada en su intersección con la estría que formó el aislado problema (Pasteran et al. 2016).

Extracción de ADN.- Para la extracción del ADN bacteriano se inocularon los aislados en Caldo Tripticasa de Soya (TSB) e incubaron a $37^{\circ} \mathrm{C}$ por 18 horas. A partir de esta suspensión se extrajo el ADN bacteriano de los aislados positivos a las pruebas fenotípicas de BLEE. Se empleó el kit comercial de extracción de ADN total Wizard ${ }^{\circledR}$ Genomic DNA Purification Kit, de la marca Promega ${ }^{\circledR}$. Se utilizó la guía emitida por el fabricante para la extracción de ADN de bacterias Gram negativas (Promega 2010).

Detección molecular de la presencia de genes de resistencia.- La detección de genes se realizó mediante reacción en cadena de la polimerasa (PCR). Los genes amplificados relacionados con la producción de BLEE fueron: bla $a_{\mathrm{TEM}} b / a_{\mathrm{CTX}-\mathrm{M}}$ y $b / a_{\mathrm{SHV}}$. Para identificar la presencia de genes relacionados a la producción de carbapenemasas se analizaron: bla $a_{\mathrm{IMP}} b / a_{\mathrm{VIM}} b / a_{\mathrm{KPC}}$ y bla $a_{\mathrm{NDM}}$.

Las reacciones de amplificación emplearon un volumen final de $25 \mu \mathrm{L}$ que contenían:12,5 $\mu \mathrm{L}$ de GoTaq $^{\oplus}$ Green Master Mix (Promega ${ }^{\mathrm{TM}}$ ); 9,5 $\mu \mathrm{L}$ de agua libre de nucleasas, $1 \mu \mathrm{L}$ de cada cebador $(10 \mu \mathrm{M})$ y $1 \mu \mathrm{L}$ de ADN $(10 \mathrm{ng} / \mu \mathrm{L})$. Las condiciones, productos y cebadores empleados en las reacciones de amplificación se detallan en la Tabla 1.

La PCR se realizó en el termociclador de gradiente MultiGene ${ }^{\mathrm{TM}}$ (modeloTC9600-G-) de la empresa LabNet Internacional. Los productos de amplificación fueron analizados por electroforesis convencional a $100 \mathrm{~V}$ por 1 hora, en geles de agarosa al $1 \%$. El buffer utilizado para los geles y la corrida electroforética fue TBE 0,5X (44,5mM Tris Base, 44,5 mM ácido bórico, 12,5mM EDTA).

Tabla 1. Cebadores utilizados en la detección de genes codificantes de Betalactamasas de Espectro Extendido (BLEE) en Enterobacterias.

\begin{tabular}{|c|c|c|c|c|c|}
\hline $\begin{array}{c}\text { Nombre del } \\
\text { Gen }\end{array}$ & $\begin{array}{l}\text { Nombre del } \\
\text { cebador }\end{array}$ & Secuencia & $\begin{array}{c}\text { Temperatura } \\
\text { de } \\
\text { anillamiento }\end{array}$ & $\begin{array}{l}\text { Amplicón } \\
\text { (pb) }\end{array}$ & Referencias \\
\hline \multirow[t]{2}{*}{ bla $\mathrm{CTX-M}$} & CTX-M F & ATAAAACCGGCAGCGGTG & $59^{\circ} \mathrm{C}$ & $483 \mathrm{pb}$ & Fatemeh et al. 2016 \\
\hline & CTX-M R & GAATTTTGACGATCGGGG & & & \\
\hline \multirow[t]{2}{*}{$b l a$ TEM } & TEM F & ATGAGTATTCAACATTTCCG & $50^{\circ} \mathrm{C}$ & $858 \mathrm{pb}$ & Ranjbar et al. 2018 \\
\hline & TEM R & CCAATGCTTAATCAGTGAGG & & & \\
\hline \multirow[t]{2}{*}{ bla $\mathrm{SHV}$} & SHV F & TTTATCGGCCYTCACTCAAGG & $55^{\circ} \mathrm{C}$ & $862 \mathrm{pb}$ & Kang et al. 2019 \\
\hline & SHV R & GCTGCGGGCCGGATAACG & & & \\
\hline \multirow[t]{2}{*}{$b^{b l a}{ }_{\mathrm{NDM}}$} & NDM F & САCСTCATGTTTGAATTCGCC & $52^{\circ} \mathrm{C}$ & $984 \mathrm{pb}$ & Dortet et al. 2012 \\
\hline & NDM R & СTCTGTCACATCGAAATCGC & & & \\
\hline \multirow[t]{2}{*}{$b^{\prime} a_{\mathrm{VIM}}$} & VIM F & ATGGTGTTTGGTCGCATATC & $52^{\circ} \mathrm{C}$ & $500 \mathrm{pb}$ & Poirel et al. 2000 \\
\hline & VIM R & TGGGCCATTCAGCCAGATC & & & \\
\hline \multirow[t]{2}{*}{$b l a_{\mathrm{IMP}}$} & IMP F & GAAGGGCGTTTATGTTCATACTT & $52^{\circ} \mathrm{C}$ & $587 \mathrm{pb}$ & Elena et al. 2018 \\
\hline & IMP R & GTTTTGCCTTACCATATTTGGA & & & \\
\hline \multirow[t]{2}{*}{$b / a_{\mathrm{KPC}}$} & KPC F & CGGAACCATTCGCTAAACTC & $55^{\circ} \mathrm{C}$ & $738 \mathrm{pb}$ & Iñiguez et al. 2010 \\
\hline & KPC R & GGCCTCGCTGTRCTTGTCAT & & & \\
\hline
\end{tabular}

$\mathrm{pb}=$ pares de bases 
Como marcador de peso molecular se utilizó Tracklt ${ }^{\mathrm{TM}} 100$ pb ADN Ladder (Invitrogen). La tinción de los geles de electroforesis obtenidos empleó SYBR ${ }^{\circledR}$ Gold 10.000X (Invitrogen). Para la visualización del gel se usó el sistema de captura de imagen Molecular Imager ${ }^{\circledR}$ Gel Doc TM $\mathrm{XR}+(\mathrm{BIO}-\mathrm{RAD})$.

Secuenciamiento de los productos de amplificación.- Los productos de amplificación que presentaron el peso molecular correspondiente al amplicón obtenido a partir del organismo control fueron enviados a la empresa MACROGEN Inc., Corea del Sur, para su purificación y secuenciamiento. Las secuencias resultantes fueron importadas al programa bioinformático Geneious Prime versión 2019.2.1 y comparadas con las secuencias obtenidas de la base de datos del National Center for Biotechnology Information (NCBI), para cada gen.

Tipificación Molecular.- La tipificación de los aislados bacterianos positivos a la producción de BLEE se realizó mediante Electroforesis en Gel de Campo Pulsado (PFGE) siguiendo los procedimientos estandarizados por los Centros para el Control y Prevención de Enfermedades (CDC) para los laboratorios de la Red Internacional PulseNet (https://www.cdc.gov/pulsenet/pdf/ ecoli-shigella-salmonella-pfge-protocol-508c. pdf). El protocolo empleado fue el recomendado para la subtificación de E. coli 0157:H7, E. coli Non-0157, Salmonella, Shigella sonnei y Shigella flexneri. El PFGE se realizó con el sistema CHEF DR-III (Bio-Rad Laboratories, Hercules, CA). La cepa Salmonella enterica serotipo Braenderup (H9812) fue utilizada como estándar de tamaño molecular para la comparación y normalización de los fragmentos obtenidos por la digestión del cromosoma bacteriano con la enzima de restricción Xba I. Los patrones de restricción fueron analizados por el software BioNumerics Seven (Applied Math, Sint-Maten-Latem, Belgium) empleando el coeficiente de similitud de DICE y el algoritmo UPGMA con 1,5\% de tolerancia y 1,5\% de optimización. Para la generación del dendrograma se aplicó un coeficiente de similaridad mayor al $90 \%$.

\section{Resultados}

Población de estudio.- De los 153 aislados bacterianos obtenidos, únicamente se seleccionaron los que presentaron el fenotipo BLEE. Con el Método de sinergia de doble disco, 14 aislados arrojaron resultados positivos y por el método automatizado VITEK $2^{\text {TM }}, 21$ fueron positivos. Se comparó los aislados positivos por cada método, y se seleccionó los que fueron positivos para al menos uno de ellos. Así, se obtuvieron 22/153 aislados con el fenotipo de interés.

De los 22 aislados positivos fenotípicamente a la producción de BLEE, el 86,4% (19/22) de los aislados fueron identificados como Escherichia coli, mientras que el 13,4\% (3/22) restante fueron individuos de la especie Klebsiella oxytoca (Tabla 2).

El origen de los aislados mostró que el 54,55 \% (12/22) de los aislados fueron obtenidos a partir de muestras de orina; $18,18 \%$ (4/22) procedieron de heridas quirúrgicas; 9,09\% (2/22) fueron obtenidos de punta de catéteres; $4,55 \%$ (1/22) se obtuvo a partir de líquido peritoneal; 4,55\% (1/22) de secreción de herida quirúrgica; 4,55 \% (1/22) de sangre y 4,55 \% (1/22) de secreción de muñón de histerectomía (Tabla 2).

Obtención de perfiles fenotípicos de resistencia.- Los valores obtenidos en las pruebas de difusión en disco complementó a los resultados reportados por el sistema automatizado VITEK $2^{\text {TM }}$ proporcionado por el centro hospitalario. Las pruebas obtenidas por el Método de difusión determinaron que el $100 \%$ (22/22) de los aislados fueron resistentes a ampicilina; $63,64 \%(14 / 22)$ fueron resistentes a ampicilina/sulbactam; 13,64\% (3/22) fueron resistentes a piperacilina/tazobactam; 90,91 \% (20/22) a cefazolina; 9,09\% (2/22) a cefoxitina; 81,82\% (18/22) a ceftriaxona; 36,36 \% (8/22) a cefepima; $13,64 \%(3 / 22)$ a gentamicina; $9,09 \%(2 / 22)$ a tobramicina; 4,55 \% (1/22) a amikacina; 72,73 \% (16/22) a ácido nalidíxico; 68,18\% (15/22) a norfloxacina; 68,18 \% (15/22) a ciprofloxacina; 63,64 \% (14/22) a levofloxacina; 54,55 \% (12/22) a trimetoprima/sulfametoxazol y 59,09\% (13/22) a aztreonam (Tabla 2).

Los resultados de los antibióticos analizados por el método automatizado VITEK $2^{\text {TM }}$ fueron: 100 $\%$ (2/2) fueron resistentes a ampicilina; 72,73 \% (16/22) a ampicilina/sulbactam; $20 \%$ (4/20) 


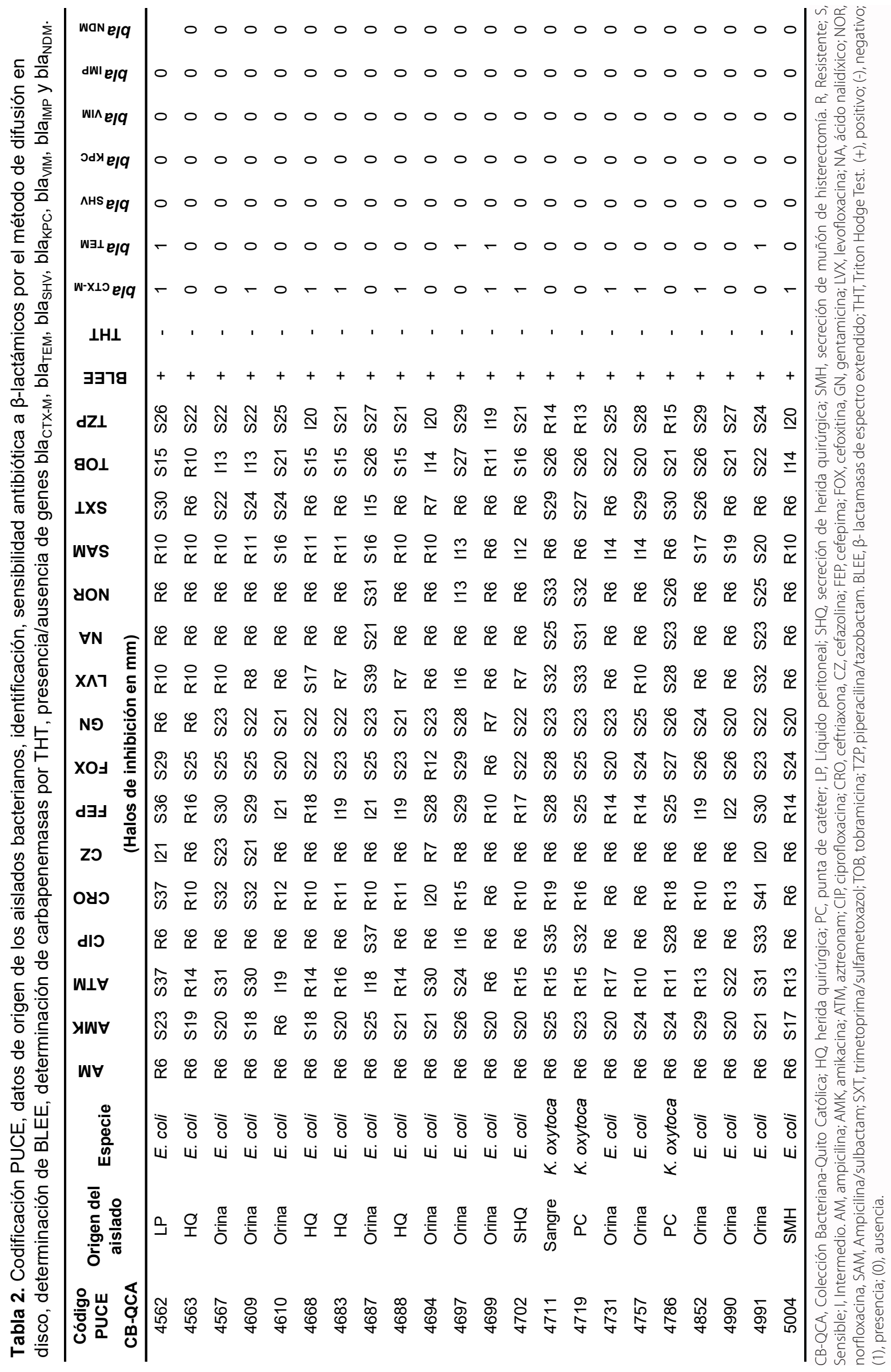


a piperacilina/tazobactam; $50 \%(1 / 2)$ a cefalotina; $50 \%(1 / 2)$ a cefuroxima; 9,09 \% (1/11) a cefoxitina; $90 \%$ (18/20) a ceftazidima; $90,48 \%$ (19/21) a ceftriaxona; 90,48 \% (19/21) a cefepima; $4,55 \%(1 / 22)$ a amikacina; 13,64 \% (3/22) a gentamicina; $72,73 \%$ (16/22) a ciprofloxacina; $50 \%$ (1/2) a norfloxacina y $50 \%$ (1/2) a trimetoprima/sulfametoxazol. Para fosfomicina, nitrofurantoina, tigeciclina y cefotaxima no se reportaron aislados resistentes a ninguno de estos antibióticos.

Identificación fenotípica de $\beta$-lactamasas de espectro extendido (BLEE).- En la prueba de sinergia de doble disco se encontró que para cefotaxima/ácido clavulánico vs cefotaxima 14/22 aislados dieron positivo a la producción de BLEE, mientras que en ceftazidima/ácido clavulánico vs. ceftazidima, 11/22 aislados dieron un resultado positivo (CLSI 2018).

El análisis por el sistema automatizado VITEK $2^{\text {TM }}$ determinó que 21 aislados presentaron el fenotipo BLEE, siendo el aislado CB-QCA 4562, el único identificado como negativo a la producción de BLEE por parte del centro hospitalario, pero con un resultado positivo por el método de sinergia de doble disco.

En relación a la detección fenotípica de la producción de carbapenemasas por el método THM, ningún aislado fue positivo a la producción de estas enzimas. En los análisis realizados por VITEK $2^{\text {TM }}$, no se detectó ningún aislado resistente a los 4 carbapenémicos probados (doripenem, ertapenem, imipenem y meropenem).

Detección molecular de la presencia de genes de resistencia.- La visualización de los productos de PCR demostró la presencia/ausencia de genes productores de BLEE y carbapenemasas. Se comparó las bandas obtenidas en los geles de electroforesis convencional con el marcador de peso molecular, buscando aquellas que coincidían con tamaño reportado para el amplicón del control positivo (Tabla 2).

De los 22 aislados analizados el $50 \%(11 / 22)$ presentó el gen bla CTX-M $_{\text {y }} 18,18 \%(4 / 22)$ el gen bla $a_{\text {TEM. }}$ Ningún aislado presentó el gen bla $a_{\mathrm{SHV}}$ (Figura 1 y Figura 2).

Ninguno de los 22 aislados estudiados dio positivo a la presencia de los 4 genes analizados para la producción de carbapenemasas: bla $a_{\mathrm{IMP}} b / a_{\mathrm{VIM}} b l a_{\mathrm{KPC}}$ y bla $a_{\mathrm{NDM}}$.
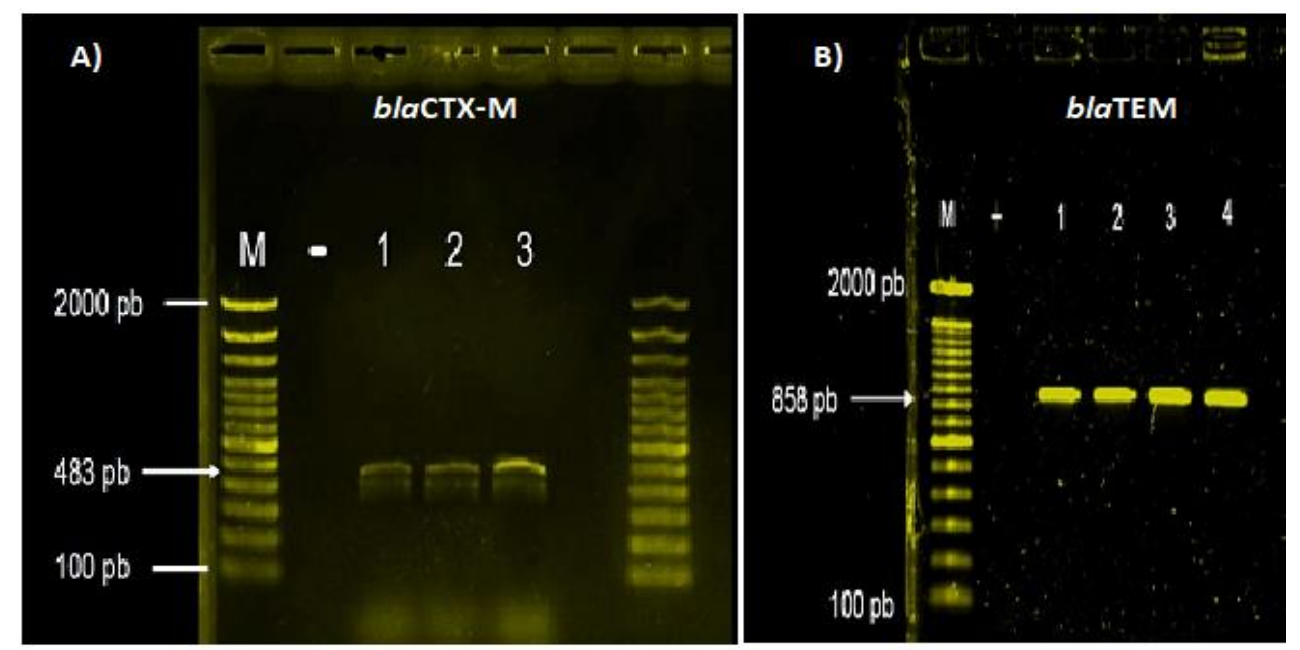

Figura 1. A) Productos de amplificación obtenidos para el gen bla ${ }_{\mathrm{CTX}-\mathrm{M} .}$ M, marcador de peso molecular (100 pb). (-), control negativo; (1), CB-QCA 4562; (2), CB-QCA 4609; (3), CB-QCA 4668. B) Productos de amplificación obtenidos para el gen bla marcador de peso molecular (100 pb). (-), control negativo; (1), CB-QCA 4562; (2), CB-QCA 4697; (3), CB-QCA 4699; (4), CB-QCA 4991. 


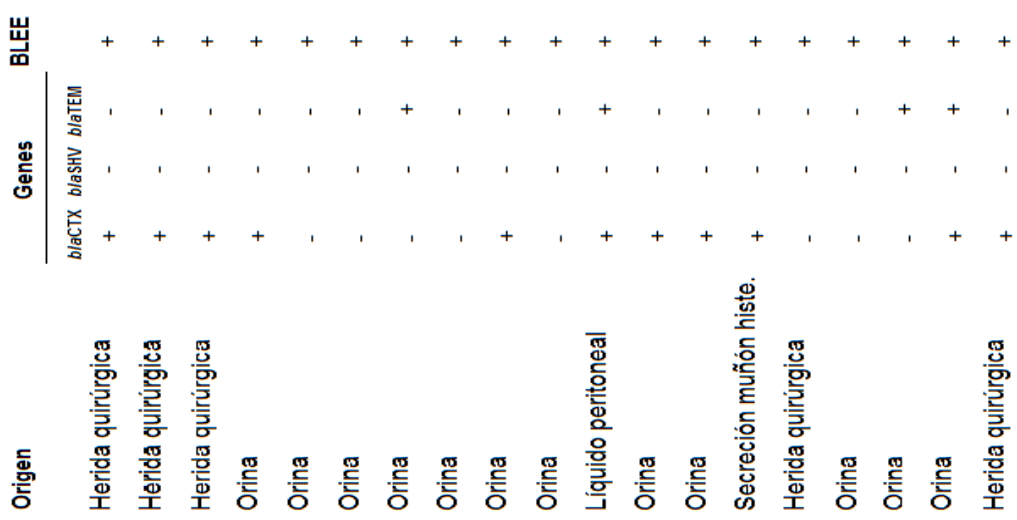

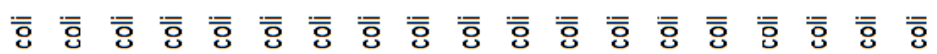

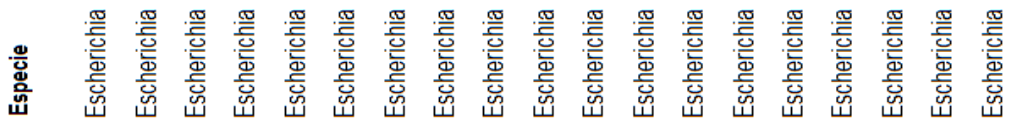

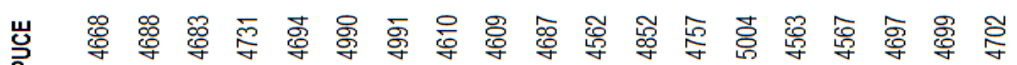

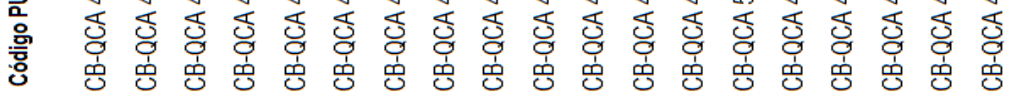

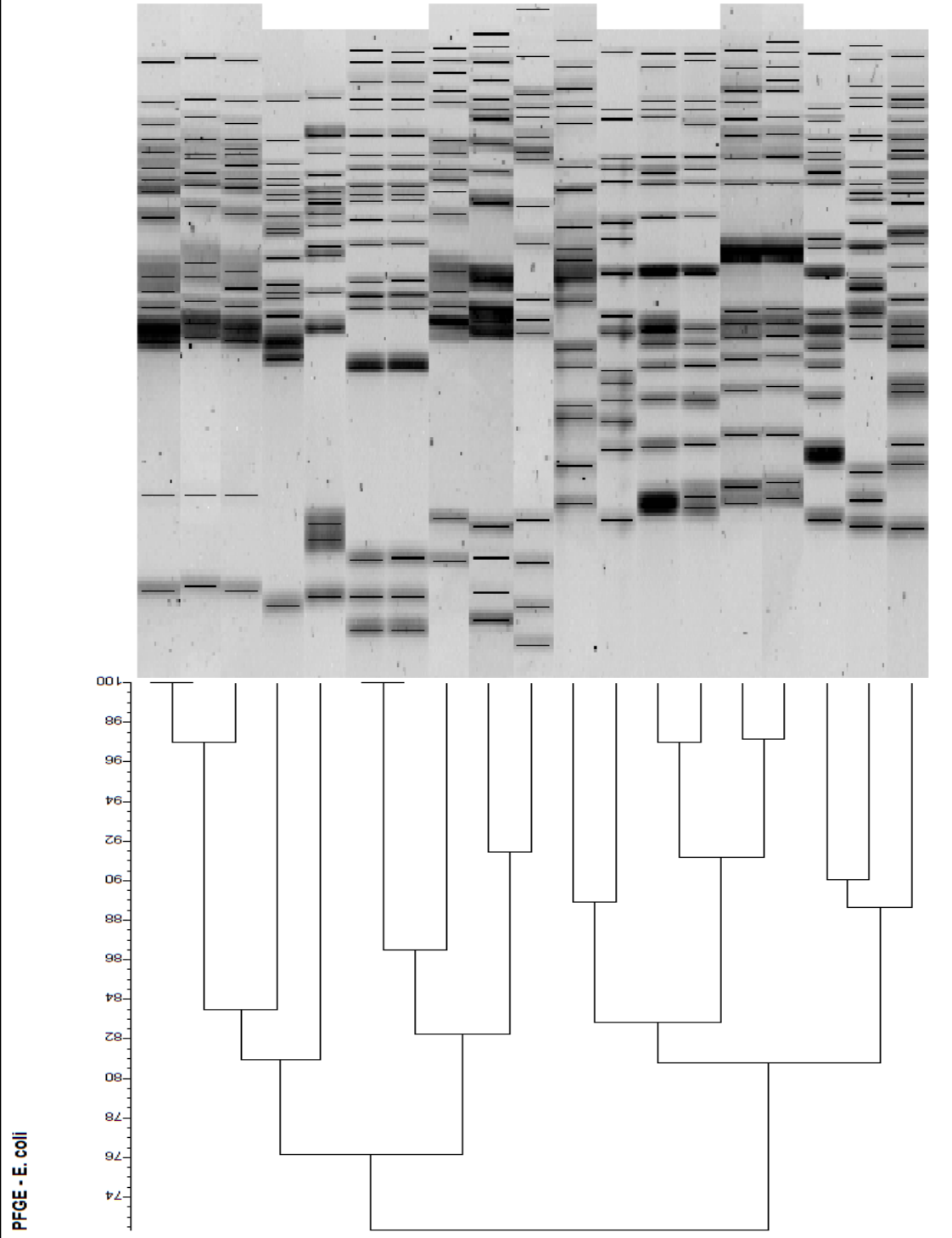

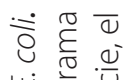

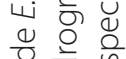
प1山 인 这峲

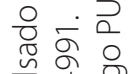

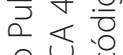
$\therefore$ 웅 है 당 $\frac{3}{4}$ Q 0 : ०० ये

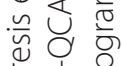
흔 吾领 递出

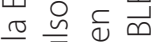

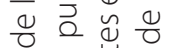

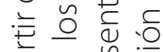
品 $>\bar{u}$ ○ $\infty$ @ nิ 은 可

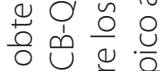
命

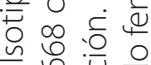

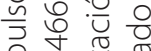
凹⿺辶, $\frac{\vec{E}}{5}$ ข 产 용요 으률요 붐 产鱼文 들. 중

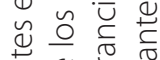

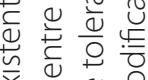

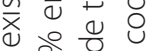

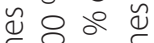

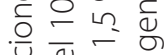

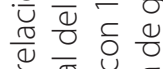

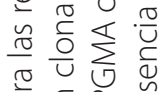
苟 :

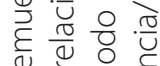
ये

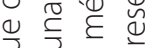
व $\overline{0} \frac{0}{0}$ है 莹

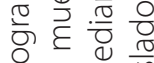
일

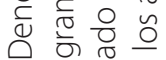
ㄴ.

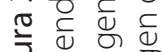
근믄 
Identificación de variantes alélicas.- La identificación se realizó comparando las secuencias de los aislados analizados con las secuencias depositadas en el NCBI de las diversas variantes alélicas. Se amplificaron 2 de los 7 genes analizados: bla $a_{\text {CTX-M }}$ y bla $a_{\text {TEM }}$. De los 22 aislados, 13 aislados presentaron al menos uno de estos genes. El gen bla $a_{\text {TEM }}$ se observó en 4 aislados y pertenecían a la variante bla $a_{\text {TEM-1 }}$ (número de accesión NG_050145.1). 11 aislados fueron portadores del gen bla $a_{\text {CTX-M }}$ y todos fueron identificados con la variante alélica bla $a_{\text {CTX-M-15 }}$ (Número de accesión NG_048935.1).

Tipificación molecular.- El PFGE realizado a los 22 aislamientos de Escherichia coli productoras de BLEE empleó un punto de corte de similitud $>90 \%$. El análisis de PFGE mostró heterogeneidad genética entre los aislados revelando 17 pulsotipos distintivos (Figura 2). Se formaron 2 clusters distintivos (grupo 1 y grupo 2) con 3 y 2 aislados respectivamente y mostraron un coeficiente de similitud del $97 \%$ y $100 \%$ cada uno. Los aislados del grupo 1 mostraron relación con la expresión de genes bla $a_{\text {CTX-M }}$ y los aislados del grupo 2 se relacionaron con la ausencia de estos genes. Los 17 pulsotipos restantes, que corresponden al 77,3\% de los aislados, mostraron coeficientes de similitud del 80 al $90 \%$ (Figura 2).

\section{Discusión}

Las bacterias productoras de $\beta$-lactamasas de espectro extendido (BLEE) son una verdadera amenaza para la sociedad, convirtiéndose en un problema mayor para los sistemas de salud a nivel mundial. El presente estudio demostró que Escherichia coli fue la especie predominante en la población estudiada, con el 86,36 \% (19/22) de aislados identificados. Este resultado coincide con otros reportes de nuestro país observados en trabajos de Calva et al. (2016), Supliguicha et al. (2017) y Pachay (2018). La predominancia de E. coli productoras de BLEE entre los aislados de origen clínico, no sólo se observa en el país, sino en varias partes del continente americano, como es el caso del estudio de Blanco et al. (2016) y Villegas et al. (2016) quienes reportan porcentajes similares de aislados de E. coli de origen clínico para Colombia, y los trabajos de Morones et al. (2016) para México.

Junto a Escherichia, el género bacteriano mayormente descrito en otros estudios de bacterias productoras de BLEE es el género Klebsiella, que ha llegado a establecerse como cepas endémicas en varios de países de América (Guzmán et al. 2014). Este estudio reportó la presencia del 13,64 $\%(3 / 22)$ de aislados relacionados con la especie Klebsiella oxytoca. Si bien, Klebsiella pneumoniae suele ser el mayor representante del género Klebsiella, estudios realizados en el país y el continente demuestran que $K$. oxytoca está presente en un porcentaje similar a K. pneumoniae, alineándose con los resultados obtenidos en esta investigación (Calva et al. 2016, Guillén et al. 2016, Morones et al. 2016, Supliguicha et al. 2017).

La mayoría de los aislados fueron obtenidos a partir de muestras de orina con el 54,55 \% (12/22) y heridas quirúrgicas con el 18,18\% (4/22). Todos los aislados fueron identificadas como Escherichia coli. La predominancia de E. coli entre los aislados procedentes de muestras de orina se debe a que $E$. coli es una bacteria comensal del sistema digestivo de todos los animales homeotermos. La cercanía anatómica entre el ano e inicio de la uretra, sobretodo en la anatomía femenina, aumenta la posibilidad de infecciones del tracto urinario (ITU) relacionados con la presencia de esta bacteria (Villar et al. 2014, Flores et al. 2015, Mathers et al. 2015). El segundo origen más común reportado fue de punta de catéter con el 9,09\% (2/22). Los 2 aislados fueron identificados como K. oxytoca. Los distintos orígenes de cada aislado se relacionan a infecciones bacterianas asociadas a la atención en salud. Estudios como el de Rivera et al. (2015) muestran la capacidad de estas dos especies, en especial de $K$. oxytoca, de sobrevivir en superficies inanimadas dentro del ambiente hospitalario. Esto explicaría el origen de su constante presencia (López, 2014, Rivera et al. 2015).

Al analizar los perfiles de resistencia obtenidos con el método de difusión en disco, las resistencias predominantes fueron a ampicilina, cefazolina y ceftriaxona. Para el método automatizado VITEK $2^{\text {TM }}$ los perfiles de resistencia predominantes fueron a ampicilina/sulbactam, ceftazidima, ceftriaxona y cefepima. Si bien, los antibióticos para los que existen un mayor porcentaje de resistencia, difieren entre un método y otro, a excepción de ceftriaxona, se observa que la 
resistencia a cefalosporinas está bien marcada. Se presenta resistencia tanto a cefalosporinas de tercera generación (ceftazidima y ceftriaxona), y cuarta generación (cefepima). Esto supone un grave problema en el tratamiento de infecciones relacionadas a bacterias con este tipo de resistencias. Las cefalosporinas son usadas como un tratamiento efectivo y de amplio espectro para tratar infecciones relacionadas con bacterias Gram positivas y Gram negativas. Además, se usan como terapia alternativa para pacientes alérgicos a las penicilinas (Macy y Contreras 2015, Oliveira et al. 2015). Su inactivación se traduce en incrementos en el uso de antibióticos reservados para casos de resistencia, como los carbapenémicos. Este problema aumenta el riesgo de generar resistencias a estas alternativas terapéuticas (Oliveira et al. 2015). Al comparar los perfiles de resistencia obtenidos con estudios similares a nivel nacional, vemos que la resistencia a ampicilina, ampicilina/sulbactam y trimetoprima/sulfametoxazol se han incrementado en gran medida en los últimos años. Estos resultados coinciden con estudios reportados en Latinoamérica por Villar et al. 2014 y Calva et al. 2016.

La producción de carbapenemasas evaluada por el Triton Hodge Test (THT) indicó que el 100 \% de los aislados analizados no presentaron producción de estas enzimas. Los resultados obtenidos por el método automatizado VITEK $2^{\text {TM }}$, mostró el mismo resultado. En el estudio planteado por Codjoe y Donkor (2017) la falta de evidencia fenotípica no significa que los aislados no sean portadores de genes relacionados a dichas resistencias. Por tanto, se analizó la presencia de genes relacionados a estas resistencias para descartar la presencia de posibles portadores.

Desde el surgimiento de las primeras bacterias productoras de BLEE, las enzimas mayormente reportadas fueron los tipos TEM y SHV. En la actualidad las enzimas predominantes corresponden al tipo CTX-M (Blanco et al. 2016). Cada una de las variantes de CTX-M está relacionado con un mayor o menor índice de resistencia a ciertos tipos de antibióticos $\beta$-lactámicos. Por este motivo, el análisis molecular de genes de resistencia es siempre necesario y complementario a los análisis fenotípicos. En el caso de esta investigación se encontró que el 50 \% (11/22) de los aislados presentaron el gen bla $a_{\mathrm{CTX}-\mathrm{M}^{\prime}}$ en específico la variante $b / a_{\mathrm{CTX}-\mathrm{M}-15}$.

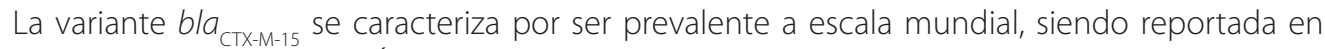
América del Norte y Sur, África, Asia y Europa. Solo en China la variante predominante es la bla $_{\text {CTX-M-14 }}$ (Chen et al. 2014, Seiffert et al. 2014, Sidjabat y Paterson 2015, Fatemeh et al. 2016). La presencia de bla CTX-M-15 $_{15}$ está en relación con los resultados documentados por Calva et al. (2016) para Ecuador y por Colquechagua et al. (2015) y Blanco et al. (2016) para Colombia y Perú respectivamente. Cantón et al. (2012) explican que la rápida propagación de este tipo de enzima BLEE se debe a la facilidad con la que puede ser transmitida al asociarse a plataformas genéticas móviles como plásmidos y transposones, lo que explicaría la predominancia de esta enzima en particular. Cantón et al. (2012) también reporta la relación de la variante bla ${ }_{C T X-M-15}$ con el plásmido Fll, Ilamado "plásmido de resistencia epidémica", caracterizado por su capacidad y facilidad de adquirir genes de resistencia (Cantón et al. 2012).

Los aislados que presentaron el gen bla ${ }_{\mathrm{CTX}-\mathrm{M}-15}$ mostraron altos porcentajes de resistencia a penicilinas, cefalosporinas (primera, segunda y tercera generación) y quinolonas. La resistencia a otro tipo de antibiótico, como las quinolonas, se relaciona con lo descrito por Flores-Mireles et al. (2015). Este estudio mostró que los genes de resistencia a quinolonas, como el gen qnrB son heredados junto a genes de resistencia a $\beta$-lactámicos como bla ${ }_{\mathrm{CTX}-\mathrm{M}-15}$ (Flores-Mireles et al. 2015). Esta relación también se observa en el estudio realizado por Azargun et al. (2018) donde se observa una gran prevalencia de resistencia a quinolonas en aislados portadores del gen bla ${ }_{\mathrm{CTX}-\mathrm{M}-15}$

Los 4 aislados de E. coli productores de la enzima TEM presentaron la variante alélica bla $a_{\text {TEM-1. }}$ La producción de la enzima TEM está relacionada con resistencia a ampicilina, penicilina y cefalosporinas. Otros estudios realizados en Ecuador demuestran que la variante bla $a_{\text {TEM-1 }}$ es la predominante en el país (Calva et al. 2016). De igual manera, el estudio de Díaz (2017), reportó que las variantes alélicas presentes fueron $b / a_{\mathrm{TEM}-1}$ y $b / a_{\mathrm{TEM}-214,}$ siendo $b / a_{\mathrm{TEM}-1}$ la predominante, resultados similares a los reportados en el presente estudio. La variante alélica obtenida bla $a_{\text {TEM-1 }}$ no es considerada como una Betalactamasa de Espectro Extendido debido a su limitada 
capacidad de hidrolizar penicilinas. Esto hace que sea catalogada como una enzima tipo Betalactamasa de Espectro Ampliado (Smet et al. 2010).

En la identificación fenotípica de BLEE, 9/22 aislados presentaron un perfil de resistencia concordante con el fenotipo, pero no presentaron ninguno de los genes codificantes de BLEE analizados. Como Rivera et al. (2015) plantea, es posible que los aislados sean portadores de otro tipo de genes relacionados con la producción de BLEE como bla $a_{\mathrm{PER}}$, bla $a_{\mathrm{VEB},}$, bla $a_{\mathrm{GES}}$, bla $a_{\mathrm{BES},}$ bla $\mathrm{T}_{\mathrm{TLA}}$ y bla ${ }_{\text {oXA }}$ (Aggeliki et al. 2014, Bajpai et al. 2017). Otra opción sugiere que los aislados que presentan el fenotipo BLEE son portadores del gen cromosómico inducible AmpC (Rivera et al. 2015).

La presencia de este gen está relacionada con la resistencia a penicilinas, cefalosporinas, cafamicinas y aztreonam. Este gen está presente en el cromosoma de algunas bacterias y también puede ser adquirido a través de elementos genéticos móviles como plásmidos (Sidjabat y Paterson 2015, Alonso et al. 2016).

En la tipificación molecular obtenida por Electroforesis en Gel de Campo Pulsado con un criterio de similitud del 90 \% se observó que no existe diseminación clonal entre los aislados analizados. Además, se pudo determinar la presencia de tres perfiles de restricción con porcentajes de similitud de 88 al $90 \%$ y dos grupos clonales con similitud del $100 \%$. Esto se traduce en la ausencia de brotes o la diseminación de un pulsotipo dominante en la población de estudio. El análisis además demostró cuatro patrones con un perfil único de restricción. Un resultado de homogeneidad se reporta comúnmente entre aislados productores de betalactamasas

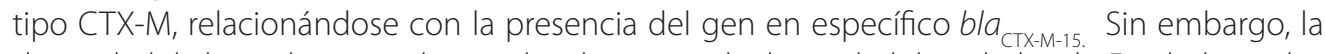
diversidad de los pulsotipos observados demuestra la diversidad de aislados de E. coli obtenidos (Izydorczyk et al. 2020).

\section{Conclusiones}

-Los aislados bacterianos de origen hospitalario obtenidos mostraron capacidad de producir $\beta$-lactamasas de espectro extendido y no carbapenemasas.

-La especie predominante en el estudio fue E. coli seguida de K. oxytoca. Este resultado concuerda con los urocultivos que fueron el origen de las muestras más común entre los aislados analizados. - Los métodos de identificación de sensibilidad antibiótica obtenida por difusión en disco y VITEK $2^{\text {TM }}$ mostraron ser precisos y efectivos.

- Los análisis moleculares caracterizaron el tipo de resistencia que se manifiesta relacionando la presencia de los genes bla $a_{\text {CTX-M-15 }}$ y bla $a_{\text {TEM-1 }}$ con la sensibilidad antibiótica obtenida.

- Los aislados positivos a la detección fenotípica de BLEE, pero no reportaron genes característicos asociados a la producción de estas enzimas estarían expresando otro tipo de genes o mecanismo de resistencia.

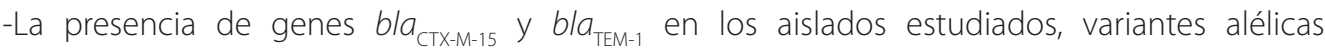
predominante a nivel mundial, denota su eficacia en la transmisión y permanencia en la población bacteriana estudiada.

-La Electroforesis en Gel de Campo Pulsado mostró la ausencia de diseminación clonal entre los aislados analizados.

\section{Agradecimientos}

Un cordial agradecimiento a la Pontificia Universidad Católica del Ecuador por el financiamiento a través de proyectos PUCE 2018, Código O13020. También al Laboratorio de Microbiología de la Escuela de Ciencias Biológicas donde se llevó a cabo el presente trabajo de investigación. A todos los integrantes del laboratorio de Microbiología quienes colaboraron de manera eficaz y desinteresada. Finalmente, un agradecimiento especial a los directivos de la Casa de Salud que colaboraron gentilmente con la donación de los aislados analizados en este estudio.

\section{Conflicto de intereses}

Los autores declaran no tener ningún conflicto de intereses.

\section{Contribución de los autores}

Duncan Coral: recolección de datos, análisis e interpretación de datos, redacción de la versión inicial del manuscrito y revisión del manuscrito. 
Iliana Alcocer: concepción y diseño del estudio, diseño experimental del estudio, revisión del manuscrito y gestión de financiamiento.

María Fernanda Yauri: concepción y diseño del estudio, diseño experimental del estudio, adquisición y análisis de aislados bacterianos, redacción de la versión inicial y final del manuscrito, revisión del manuscrito y gestión de financiamiento.

\section{Referencias bibliográficas}

Adeolu M, Alnajar S, Naushad S, Gupta R. 2016. Genome-based phylogeny and taxonomy of the 'Enterobacteriales': Proposal for enterobacterales ord. nov. divided into the families Enterobacteriaceae, Erwiniaceae fam. nov., Pectobacteriaceae fam. nov., Yersiniaceae fam. nov., Hafniaceae fam. nov., Morgane. Int J Syst Evol Microbiol. 66(12):5575-5599.

Aggeliki P, Evgenia G, Georgia V, Vassiliki K, Theodoros P, Spyros P, Athanassios T. 2014. Test for Phenotypic Detection of ESBLs among Enterobacteriaceae Producing Various $\beta$-Lactamases. J Clin Microbiol. 52(5):1483-1489.

Alonso N, Miró E, Pascual V, Rivera A, Simó M, Garcia MC, Xercavins M, Morera MA, Espejo E, Gurguí M, et al. 2016. Molecular characterisation of acquired and overproduced chromosomal blaAmpC in Escherichia coli clinical isolates. Int J Antimicrob Agents. 47(1):62-68.

Azargun R, Sadeghi M, Hossein M, Barhaghi S, Kafil H, Yeganeh F, Oskouee M, Ghotaslou R. 2018. The prevalence of plasmid-mediated quinolone resistance and ESBL-production in enterobacteriaceae isolated from urinary tract infections. Infect Drug Resist. 11:1007-1014.

Bajpai T, Pandey M, Varma M, Bhatambare G. 2017. Prevalence of TEM , SHV , and CTX - M Beta - Lactamase genes in the urinary isolates of a tertiary care hospital. Avicenna J Med. 7(1):12-16.

BD Diagnostics. 2009. Difco and BBL Manual Manual of Microbiological Culture Media 2nd Edition.

Bauer A, Kirby W, Sherris J, TurckM. 1966. Antibiotic Susceptibility Testing By A Standardized Single Disk Method. Am J Clin Pathol. 45(4):493-496.

Bevan E, Jones A, Hawkey P. 2017. Global epidemiology of CTX-M $\beta$-lactamases: Temporal and geographical shifts in genotype. J Antimicrob Chemother. 72(8):2145-2155.

Blanco V, Maya J, Correa A, Perenguez M, Muñoz J, Motoa G, Pallares C, Rosso F, Matta L, Celis Y, et al. 2016. Prevalencia y factores de riesgo para infecciones del tracto urinario de inicio en la comunidad causadas por Escherichia coli productor de betalactamasas de espectro extendido en Colombia. Enferm Infecc Microbiol Clin. 34(9):559-565.

Brolund A, Sandegren L. 2016. Characterization of ESBL disseminating plasmids. Infect Dis (Auckl). 48(1):18-25.

Calva D, Toledo Z, Ochoa S, Arévalo A, Ausili A. 2016. Detection and molecular characterization of $\beta$-lactamase genes in clinical isolates of Gram-negative bacteria in Southern Ecuador. Brazilian J Infect Dis. 20(6):627-630.

Cantón R, González-Alba J, Galán J. 2012. CTX-M enzymes: Origin and diffusion. Front Microbiol. 3(APR).

(CDC) Centers for Disease Control and Prevention. 2013. Vital Signs: Carbapenem-Resistant Enterobacteriaceae. MMWR Morb Mortal Wkly Rep. 62(9):165-170.

Chen L, Freeman J, Nicholson B, Keiger A, Lancaster S, Joyce M, Woods C, Cook E, Adcock L, Louis S, et al. 2014. Widespread dissemination of CTX-M-15 genotype extended-spectrum- $\beta$ - 
lactamase-producing enterobacteriaceae among patients presenting to community hospitals in the southeastern United States. Antimicrob Agents Chemother. 58(2):1200-1202.

Cho H, Uehara T, Bernhardt T. 2014. Beta-lactam antibiotics induce a lethal malfunctioning of the bacterial cell wall synthesis machinery. Cell. 159(6):1300-1311.

(CLSI) Clinical and Laboratory Standards Institute. 2018. Clinical and Laboratory Standards Institute antimicrobial susceptibility testing standards M02, M07, and M11.

Codjoe F, Donkor E. 2017. Carbapenem Resistance: A Review. Med Sci. 6(1):1.

Colquechagua F, Sevilla C, Gonzales E. 2015. Artículo Original de espectro extendido en muestras fecales en el enterobacteriaceae in fecal samples at the national. Rev Peru Med Exp Salud Publica. 32(1):26-32.

D’Andrea M, Arena F, Pallecchi L, Rossolini G. 2013. CTX-M-type $\beta$-lactamases: A successful story of antibiotic resistance. Int J Med Microbiol. 303(6-7):305-317.

Díaz V. 2017. Caracterización molecular de genes de resistencia a $\beta$-lactámicos en aislados clínicos de Escherichia coli provenientes de urocultivos y pruebas de inhibición con péptidos de Boana rosenbergi y Rana sp. [tesis]. [Quito (UIO)]: Pontificia Universidad Católica del Ecuador.

Ding H, Liu B, Gao Y, Zhong X, Duan S, Yuan L. 2018. Divergence of affinities, serotypes and virulence factor between CTX-M Escherichia coli and non-CTX-M producers. Poult Sci. 97(3):980985.

Doi Y, lovleva A, Bonomo RA. 2017. The ecology of extended-spectrum $\beta$-lactamases (ESBLs) in the developed world. J Travel Med. 24(1):S44-S51.

Dortet L, Nordmann P, Poirel L. 2012. Association of the Emerging Carbapenemase NDM-1 with aBleomycin Resistance Protein inEnterobacteriaceaeandAcinetobacter baumannii. Antimicrobial agents and chemotherapy. 56(4):1693.

Elena A, Cejas D, Magariños F, Jewtuchowicz V, Facente A, Gutkind G, Di Conza J, Radice M. 2018. Spread of Clonally RelatedEscherichia coli Strains Harboringan IncA/C1 Plasmid Encoding IMP8 and Its Recruitment intoan Unrelated MCR-1-Containing Isolate. Antimicrobial agents and chemotherapy. 62(6): 02414-02417.

Fatemeh F, Hamed A, Ali H, Hossein G, Latif G, Parastoo H. 2016. Antibiotic susceptibility patterns in CTX-M-15-producing Enterobacteraceae isolated from healthy Afghan refugees in Iran. African J Microbiol Res. 10(11):357-362.

Flores A, Walker J, Caparon M, Hultgren SJ. 2015. Urinary tract infections: Epidemiology, mechanisms of infection and treatment options. Nat Rev Microbiol. 13(5):269-284.

Guillén, R. et al. 2016. Detección molecular de belactamasas de espectro extendido (BLEE) en enterobacterias aisladas en Asunción. Mem Inst Investig Cienc Salud. 14(1):8-16.

Guzmán-Blanco M, Labarca J, Villegas M, Gotuzzo E. 2014. Extended spectrum $\beta$-lactamase producers among nosocomial Enterobacteriaceae in Latin America. Brazilian J Infect Dis. 18(4):421-433.

Iñiguez D, Zurita J, Alcocer I, Ortega D, Gómez AM, Maldonado L. 2012. Klebsiella pneumoniae productora de carbapenemasa tipo KPC-2: primer reporte en el Ecuador. Rev la Fac Ciencias Médicas. 37(1-2):40-43.

INEC (2017). Programa Nacional de Estadística 2017-2021. Instituto Nacional de Estadística y Censos, Quito-Ecuador. 
Izydorczyk C, Waddell B, Edwards B, Greysson-Wong J, Surette M, Somayaji R, Rabin H, Conly J, Church D, \& Parkins M. 2020. Epidemiology of E. coli in Cystic Fibrosis Airways Demonstrates the Capacity for Persistent Infection but Not Patient-Patient Transmission. Frontiers in microbiology, 11, 475. https://doi.org/10.3389/fmicb.2020.00475

López L. 2014. Papel del ambiente hospitalario y los equipamientos en la transmisión de las infecciones nosocomiales. Enferm Infecc Microbiol Clin. 32(7):459-464.

López D, Torres M, Prada C. 2016. Genes de resistencia en bacilos Gram negativos: Impacto en la salud pública en Colombia. Univ y Salud. 18(1):190.

Macy E, Contreras R. 2015. Adverse reactions associated with oral and parenteral use of cephalosporins: A retrospective population-based analysis. J Allergy Clin Immunol. 135(3):745752.e5.

Mathers A, Peirano G, Pitout J. 2015. The role of epidemic resistance plasmids and international high- risk clones in the spread of multidrug-resistant Enterobacteriaceae. Clin Microbiol Rev. 28(3):565-591.

Morones I, Salgado T, Gonzaga T, Matamoros A, Terán J, Arteaga S, Castro L, Reyes A, López D, Meza D. 2016. Enterobacterias con betalactamasas de espectro extendido en hemocultivos y urocultivos. Med Interna Mex. 32(4):381-387.

Numanovic F, Hukic M, Delibegovic Z, Tihic N, Pasic S. 2013. Comparison of double disk synergy test, VITEK 2 and Check- MDR CT102 for detection of ESBL producing isolates. Acta Medica Acad 2013. 42(1):15-24.

Oliveira M, Oliveira C, Gonçalves K, Santos M, Tardelli A, Nobre V. 2015. Enterobacteriaceae resistant to third generation cephalosporins upon hospital admission: Risk factors and clinical outcomes. Brazilian J Infect Dis. 19(3):239-245.

Kang H, Wang L, Li Y, Lu Y, Fan W, Bi R, Qian H, Gu B. 2019. Dissemination of Multidrug-Resistant Shigella flexneri and Shigella sonnei with Class 1, Class 2, and Atypical Class 1 Integrons in China. Microb Drug Resist. 00(00):mdr.2018.0229.

Organización Mundial de la Salud (OMS) [Internet]. 2018. Bangkok. Datos recientes revelan los altos niveles de resistencia a los antibióticos en todo el mundo; [updated 2018 Ene 30; cited 2020 Nov 20]. Disponible en: https://www.who.int/es/news/item/29-01-2018-high-levels-ofantibiotic-resistance-found-worldwide-new-data-shows

Pachay S. 2018. Las infecciones bacterianas y su resistencia a los antibióticos. Caso de estudio: Hospital Oncológico “Dr. Julio Villacreses Colmont Solca”, Portoviejo. Univ y Soc. 10(3):134-141.

Pasteran F, Gonzalez L, Albornoz E, Bahr G, Vila A, Corso A. 2016. Triton Hodge test: improved protocol for modified Hodge test for enhanced detection of NDM and other carbapenemase producers. J Clin Microbiol 54:640 -649. doi:10.1128/JCM.01298-15.

Poirel L, Naas T, Nicolas D, Collet L, Bellais S, Cavallo J, Nordmann P. 2000. Characterization of VIM-2, a Carbapenem-Hydrolyzing Metallo- $\beta$-Lactamase and Its Plasmid and Integron Borne Gene from a Pseudomonas aeruginosa Clinical Isolate in France. Antimicrobial agents and chemotherapy. 44: 891-897.

Promega C. 2010. Isolation of Genomic DNA from Whole Blood Wizard ${ }^{\circledR}$ Genomic DNA Purification Kit. :1123-1126.

Ranjbar R, Ardashiri M, Samadi S, Afshar D. 2018. Distribution of extended-spectrum $\beta$-lactamases (ESBLS) among salmonella serogroups isolated from pediatric patients. Iran J Microbiol. 10(5):294-299. 
Rivera M, Rodriguez C, Flores R, Serquén L, Arce Z. 2015. Betalactamasas De Espectro Extendido Tipo Tem. Rev Peru Med Exp Salud Publica. 32(4):752-755.

Rivoarilala OL, Garin B, Andriamahery F, Collard JM. 2018. Rapid in vitro detection of CTX-M groups 1, 2, 8, 9 resistance genes by LAMP assays. PLoS One. 13(7):1-15.

Rossolini G, Andrea M, Mugnaioli C. 2008. The spread of CTX-M-type extended-spectrum betalactamases. 14:33-41.

Seiffert S, Marschall J, Perreten V, Carattoli A, Furrer H, Endimiani A. 2014. Emergence of Klebsiella pneumoniae co-producing NDM-1, OXA-48, CTX-M-15, CMY-16, QnrA and ArmA in Switzerland. Int J Antimicrob Agents. 44(3):260-262.

Shah K, Shrimali G, Mulla S. 2016. Comparison of double disc diffusion method and VITEK 2 compact system to screen the esbl producers in intensive care unit in hospital. 7(9):7-9.

Shaikh S, Fatima J, Shakil S, Rizvi S, Kamal M. 2015. Antibiotic resistance and extended spectrum beta-lactamases: Types, epdemiology and treatment. Saudi J Biol Sci. 22(1):90-101.

Sidjabat H, Paterson D. 2015. Multidrug-resistant Escherichia coli in Asia: Epidemiology and management. Expert Rev Anti Infect Ther. 13(5):575-591.

Smet A, Martel A, Persoons D, Dewulf J, Heyndrickx M, Herman L, Haesebrouck F, Butaye P. 2010. Broad-spectrum $\beta$-lactamases among Enterobacteriaceae of animal origin: Molecular aspects, mobility and impact on public health. FEMS Microbiol Rev. 34(3):295-316.

Supliguicha M, Supliguicha P, Ortega V, Pacurucu C, Lema J, Santander P, Delgado C, León V, Bermeo $H$, Peñafiel $E$, et al. 2017. Factores de riesgo para la infección del tracto urinario por enterobacterias productoras de betalactamasas de espectro extendido Resumen Risk factors for infection of urinary tract by extended-spectrum beta-lactamase producing enterobacteriaceae. 36.

Villar H, Jugo M, Macan A, Visser M, Hidalgo M, Maccallini G. 2014. Frequency and antibiotic susceptibility patterns of urinary pathogens in male outpatients in Argentina. J Infect Dev Ctries. 8(6):699-704.

Villegas M, Pallares C, Escandón K, Hernández C, Correa A, Álvarez C, Rosso F, Matta L, Luna C, Zurita J, et al. 2016. Characterization and clinical impact of bloodstream infection caused by carbapenemase-producing enterobacteriaceae in seven Latin American countries. PLoS One. 11(4):1-13.

Woerther P, Andremont A, Kantele A. 2017. Travel-acquired ESBL-producing Enterobacteriaceae: impact of colonization at individual and community level. J Travel Med. 24(1):S29-S34.

Yigit H, Queenan A, Anderson G, Domenech A, Biddle J, Steward C. 2001. Novel CarbapenemHydrolyzing $\beta$-Lactamase, KPC-1, from a Carbapenem-Resistant Strain of. Society. 45(4):11511161.

Zeng X, Lin J. 2013. Beta-lactamase induction and cell wall metabolism in Gram-negative bacteria. Front Microbiol. 4(MAY):1-9.

Zurita J, Alcocer I, Ortega-paredes D, Barba P, Yauri F. 2013. Carbapenem-hydrolysing $\beta$-lactamase KPC-2 in Klebsiella pneumoniae Carbapenem-hydrolysing b-lactamase KPC-2 in Klebsiella pneumoniae isolated in Ecuadorian hospitals. J Glob Antimicrob Resist. 1(February 2018):6-8. 